\title{
Implementing Flywheel (Isoinertial) Exercise in Strength Training: Current Evidence, Practical Recommendations, and Future Directions
}

\author{
Marco Beato $^{1 *}$ and Antonio Dello lacono ${ }^{2}$ \\ ${ }^{1}$ School of Health and Sports Sciences, University of Suffolk, Ipswich, United Kingdom, ${ }^{2}$ Institute of Clinical Exercise and \\ Health Science, School of Health and Life Sciences, University of the West of Scotland, Hamilton, United Kingdom
}

Keywords: flywheel, training, PAPE, performance, strength

\section{INTRODUCTION}

The concept of isoinertial training using flywheel devices has been developed in the recent past with the first evidence supporting its efficacy as conditioning method only dating back to the early

\section{OPEN ACCESS}

Edited by:

Luca Paolo Ardigò,

University of Verona, Italy

Reviewed by:

Sergio Maroto-Izquierdo, Miguel de Cervantes European University, Spain Rodrigo Fernandez-Gonzalo, Karolinska Institutet, Sweden

*Correspondence: Marco Beato M.Beato@uos.ac.uk

Specialty section:

This article was submitted to

Exercise Physiology,

a section of the journal

Frontiers in Physiology

Received: 12 February 2020

Accepted: 07 May 2020

Published: 03 June 2020

Citation:

Beato M and Dello lacono A (2020)

Implementing Flywheel (Isoinertial)

Exercise in Strength Training: Current

Evidence, Practical

Recommendations, and Future

Directions. Front. Physiol. 11:569.

doi: $10.3389 /$ fphys.2020.00569 1990's (Colliander and Tesch, 1990; Dudley et al., 1991). Flywheel exercises were initially proposed to mitigate the neuromuscular dysfunctions and concurrent muscle atrophy of the musculoskeletal system in astronauts caused by the absence of gravity during long-duration space travels (Dudley et al., 1991; Berg and Tesch, 1994; Norrbrand et al., 2008). Since then, many studies have described the mechanical advantages of the flywheel devices and attempted to clarify the neurophysiological mechanisms, morphological adaptations and training effects induced by flywheel exercise as both acute and chronic conditioning strategies (Maroto-Izquierdo et al., 2017; Tesch et al., 2017; Beato et al., 2019d). The preliminary and promising evidence has inherently fostered increasing interest among sport science researchers and applied practitioners toward the potential and beneficial implementation of flywheel exercises in the fields of athletic performance development, injury prevention, and clinical rehabilitation (Tous-Fajardo et al., 2006, 2016; de Hoyo et al., 2015; Tesch et al., 2017; Beato et al., 2019a). However, in spite of the growing use of flywheel exercises in the last few years, there is still a gap in the literature providing precise recommendations on how to accurately design and prescribe flywheel exercises using a systematic approach especially in elite sport athletes (Maroto-Izquierdo et al., 2017; Beato et al., 2019c; Franchi and Maffiuletti, 2019). In light of the contemporary scientific evidence, the purpose of this commentary is to provide precise recommendations about flywheel training to enhance sports performance, thus facilitating an informed implementation of this conditioning method in research and applied settings.

\section{Physiology of Eccentric Exercises}

Extensive research has been conducted on eccentric resistance training applications with an overall support of its utilization to induce positive adaptations in both untrained populations and sports athletes (Wernbom et al., 2007; Roig et al., 2009; Wagle et al., 2017; Suchomel et al., 2018; Franchi and Maffiuletti, 2019).

At molecular level, eccentric exercises may induce a preferential upregulation of satellite cell activity and transcriptional pathways in fast-twitch muscle fibers, being these fibers the most damaged during the eccentric contraction (Moore et al., 2005; Toigo and Boutellier, 2006; Cermak et al., 2013). Moreover, it has been documented that protein synthesis can be further increased when both force generation and musculotendinous stretch occur concurrently, which are both involved 
during the eccentric contraction. These molecular responses presumably represent the mechanisms that increase the net muscular protein accretion underpinning hypertrophic effects and increases of muscle fiber cross-sectional area (CSA) (Douglas et al., 2017, 2018; Hody et al., 2019).

From a mechanical perspective, during eccentric contractions, muscles act while lengthening due to the applied external resistance which exceeds the momentary force produced by the muscle (Douglas et al., 2017). This mechanical advantage also represents the rationale for using flywheel exercises in which the eccentric phase results overloaded by the inertia accumulated during the concentric phase at condition that the latter is executed at maximal effort (Maroto-Izquierdo et al., 2017). Specifically, a few advantageous physiological effects arise from these augmented eccentric muscle actions. First, eccentric contraction is accompanied by a higher force output production and lower energy expenditure compared with both isometric and concentric muscle contractions, thus resulting in greater work efficiency (Zamparo et al., 2015; Douglas et al., 2017). These outputs are attributed to the higher number of attached cross-bridges and by the tensile contribution of the passive structure elements engaged within the sarcomere under elongation/lengthening (Douglas et al., 2017; Hody et al., 2019). The eccentric contraction also favors specific neural patterns such as fewer motor units required to generate the same amount of force during a submaximal exercise (Douglas et al., 2017). Moreover, eccentric actions allow for preferential recruitment of high threshold motor unit and greater cortical activity (Hody et al., 2019). Finally, the eccentric training may improve the kinetic efficiency of the musculotendinous unit, in fact, the repeated bouts of eccentric actions cause structural damage at muscle level where the overstretched sarcomeres become progressively weaker and disrupted (Hody et al., 2019). The disruption of sarcomeres along muscle fibers leads to an increase of series compliance which is known as a primary mechanism of the enhanced muscle mechanical outputs (Douglas et al., 2017; Hody et al., 2019). These physiological peculiarities support the advantageous use of flywheel training to optimize acute and chronic adaptations.

\section{Acute Responses and Post-Activation Potentiation Enhancement (PAPE) Effects}

PAPE refers to an acute enhancement of athletic performance following a pre-load activity (Blazevich and Babault, 2019; Dello Iacono et al., 2019; Wallace et al., 2019). The most supported mechanism underpinning PAPE is related to the phosphorylation of the myosin regulatory light chain, which leads to an greater force and rate of force development during muscle contractions (Tillin and Bishop, 2009; Boullosa et al., 2018). PAPE using traditional resistance exercises has been extensively investigated, while evidence supporting flywheel exercises for such a purpose is still limited (Cuenca-Fernández et al., 2018; Beato et al., 2020). These preliminary findings suggest implementing flywheel exercises to elicit PAPE thus pointing to them as a viable alternative to traditional protocols (Cuenca-Fernández et al., $2015,2019)$. However, no evidence is currently available as to what conditioning activity may be advantageous or superior in enhancing athletic performances (Beato et al., 2019a). The rationale for utilizing flywheel exercises to facilitate PAPE effects stems from the advantageous neuromuscular responses and mechanical adaptations elicited by the augmented eccentric muscle actions such as greater motor unit discharge rates in conjunction with possible selective recruitment of higher-order motor units and improved synchronization (de Hoyo et al., 2014; Beato et al., 2019d). Moreover, the ability to fully and effectively activate muscle increases as a factor of the eccentric contraction velocity and more interestingly is concurrently accompanied by greater force outputs (Franchi and Maffiuletti, 2019). During eccentric contractions, force output increases with velocity up to a certain point, after which it levels off or decays slightly. Great force outputs developed at higher velocities consequently result in increased muscular power responses, which is a further advantageous effect of flywheel exercises as potentiating activities (Douglas et al., 2017). Nevertheless, very few studies have investigated the underpinning acute neuromuscular mechanisms and associated acute musculoskeletal adaptations induced by flywheel exercises and their acute effect on PAPE (Beato et al., 2019c). Therefore, future research is needed to clarify these aspects before providing informative guidelines.

\section{Chronic Adaptations and Long-Term Training Effects}

The unique neural patterns and mechanical responses elicited by the combination of both concentric and overloaded eccentric contractions of flywheel exercises have also important implications for the chronic adaptations they may induce when implemented in the form of long-term training (MarotoIzquierdo et al., 2017; Nuñez Sanchez and Sáez de Villarreal, 2017; Petré et al., 2018). In particular, flywheel training is a valid method leading to positive morphological changes of the muscle structure and architecture, and mechanical adaptations like hypertrophy effects and strength gains, respectively (MarotoIzquierdo et al., 2017). Previous evidence reported that the eccentric overload per se may not be sufficient to stimulate muscle mass growth (Wernbom et al., 2007), whereas the hypertrophic benefits offered by flywheel training likely stem from the combination of concentric-eccentric contractions (Norrbrand et al., 2008; Nuñez Sanchez and Sáez de Villarreal, 2017). In fact, the maximal effort athletes exploit during the concentric phase of flywheel exercises is the required conditio sine qua non whereby an effective eccentric overload can occur. Nevertheless, the characteristic eccentric overload of flywheel exercises is considered the necessary stimulus leading to greater strength gains over resistance training protocols including only concentric exercises (Nuñez Sanchez and Sáez de Villarreal, 2017). A previous meta-analysis reported that significant increases in muscle volume and CSA can be expected following 5-8 weeks of flywheel training (Petré et al., 2018). Flywheel training over a period of 10-11 weeks was also effective to enhance soccerspecific tasks such as jump, linear sprint and change of direction capabilities (de Hoyo et al., 2015; Tous-Fajardo et al., 2016). Additionally, flywheel training may also be implemented in 
injury prevention, due to the protective role of the emphasized eccentric component particularly for the hamstring muscles (Askling et al., 2003; de Hoyo et al., 2015). This assumption is supported by previous evidence reporting 10 weeks of flywheel training including 1-2 weekly sessions to reduce hamstring muscle injury incidence and severity in elite-level young soccer players (de Hoyo et al., 2015). In summary, the benefits offered by flywheel exercises are mainly based on the common physiological and mechanical background of eccentric contractions, which lead to beneficial neuromuscular, morphological, and function adaptations underpinning performance enhancements in athletic tasks like vertical jumps, sprints, and changes of direction (Maroto-Izquierdo et al., 2017; Suchomel et al., 2018; Coratella et al., 2019).

\section{Informed Implementation of Acute Flywheel Exercise Protocols in Research Settings and Applied Contexts}

Although the scientific evidence on the PAPE effects of flywheel exercises is relatively limited, a few practical recommendations can be provided for the correct methodological implementation of flywheel exercises as PAPE protocols.

(a) Training intensity: a broad range of inertial intensities $\left(0.029-0.11 \mathrm{~kg} \cdot \mathrm{m}^{2}\right)$ can be confidently used to enhance the subsequent sport-specific tasks, such as vertical and horizontal jumps and change of direction (de Hoyo et al., 2014; Beato et al., 2019a,b, 2020).

(b) Training volume: multiple sets of flywheel exercises induce superior PAPE effects compared to single set protocols. Therefore, protocols designed as multiple sets (e.g., 2-3 sets) are recommended to obtain acute enhancements on athletic performances (Beato et al., 2020; de Keijzer et al., 2020).

(c) Rest interval: The time-course of the flywheel exercise PAPE effects seems to be consistent with the gravitational loadingbased PAPE literature (Beato et al., 2020). An acute decrease in performance is observed up to $3 \mathrm{~min}$, whereas PAPE is dominant in the minutes thereafter and potentially lasting up to $9 \mathrm{~min}$ (Beato et al., 2019d). This should be taken into account when planning the rest period between the conditioning stimulus and subsequent activity.

(d) Training Specificity: Exercise specificity and similarity between the flywheel PAPE protocol and the subsequent athletic tasks may have importance for exploiting optimal PAPE effects (Cuenca-Fernández et al., 2018). However, there is no definitive evidence in support of this theory since contrasting findings exist (Beato et al., 2019c), thus future research is warranted to verify such a hypothesis.

(e) Familiarization: The relatively greater mechanical demands of flywheel exercise require a minimum of 2-3 familiarization to become acquainted with this training method and to optimize its implementation as PAPE protocols (Sabido et al., 2018; Hody et al., 2019). Familiarization is also important to optimize chronical adaptations since specific eccentric strategies are needed when applying braking forces at the desired joints' angles and throughout the range of motion to obtain an eccentric overload (e.g., athletes may voluntarily delay the braking action) (Tous-Fajardo et al., 2006).

\section{Informed Implementation of Chronic Flywheel Exercise Protocols in Research Settings and Applied Contexts}

In light of the observed beneficial chronic adaptations of flywheel training on muscle strength, power and hypertrophy with consequent training effects on vertical jumps, sprints and change of direction (Maroto-Izquierdo et al., 2017), the following evidence-based guidelines can be provided:

(a) Training intensity: a range of inertial intensities (0.05-0.11 $\mathrm{kg} \cdot \mathrm{m}^{2}$ ) are generally recommended to induce chronical adaptations and enhance the athletic performances (SuarezArrones et al., 2018; Coratella et al., 2019; Maroto-Izquierdo et al., 2019). Higher inertial intensities may be preferable to develop force, while lower inertial intensities could be used for power purposes (Martinez-Aranda and FernandezGonzalo, 2017). However, limited evidence is available about the optimal inertial load required to selectively maximize chronic effects across medium and long training periods.

(b) Training volume: as consistently reported in the literature, the protocols using multiple sets (from 3 to 6) and repetitions (from 6 to 8 ) are reasonably implementable to ensure chronic adaptations and performance enhancements outputs (Maroto-Izquierdo et al., 2017; Coratella et al., 2019).

(c) Training frequency and duration: although clear guidelines about frequency and duration aspects of flywheel training are missing, 2-3 sessions per week completed for 5-10 weeks appear sufficient for inducing positive adaptive effects (Maroto-Izquierdo et al., 2017; Coratella et al., 2019). Recently, positive effects on change of direction, sprinting and jumping capabilities were observed following an 8-week training program completed as only 1 session per week, but evidence confirming such findings has to be produced yet (Coratella et al., 2019). Additionally, there is evidence that early functional and morphological adaptations can be obtained following short-term (4 weeks) flywheel-squat protocols (5 sets of 10 repetitions) (IlleraDomínguez et al., 2018).

\section{FUTURE DIRECTIONS}

From the existing literature a few questions emerge which should be acknowledged and discussed in view of future research directions (Figure 1):

(a) Elite athletes and females: few studies have enrolled professional adult team-sport or female athletes with consequent uncertainty about the beneficial application of flywheel training to enhance athletic performances in these populations (Fernandez-Gonzalo et al., 2014; TousFajardo et al., 2016; Maroto-Izquierdo et al., 2017). Age, gender, strength levels, training history, and motivation 


\section{Current evidence, practical recommendations and future research directions of flywheel training}

\section{Advantages \\ Limitations}

a) eccentric contractions

b) Higher force and power production combined with lower energy expenditure

c) Preferential recruitment of high threshold motor unit and greater cortical activity

d) Evidence supporting flywheel exercises for PAPE purposes

e) Evidence supporting flywheel training to induce chronic adaptations on muscular strength, power and hypertrophy

f) Evidence supporting flywheel training to improve athletic performances (jumping, changes of direction, and sprinting performance) a) Familiarization process required

b) Lack of standard procedures for exercise loading prescription and monitoring (encoder needed)

c) Limited evidence for elite sport athletes

d) Not clear superiority compared to traditional resistance training methods for both acute and chronic effects

e) Few high-quality RCT published

f) Limited use in clinical settings due to the maximal concentric effort required to generate overload alongside proper technique of execution

\section{Future directions}

a) High-quality RCT involving elite sports and female athletes

b) Needed guidelines about flywheel training variables (exercise type, volume, intensity, rest interval, frequency, duration) for PAPE and chronical purposes

c) Needed guidelines about training loading prescription and monitoring procedures

d) Comparative investigations with traditional resistance exercises of long duration $(>10$ weeks)

e) Dose-response of flywheel training in professional athletes

FIGURE 1 | PAPE, post-activation potentiation enhancement; RCT, Randomized controlled trial.

all appear to influence the concentric and eccentric forcevelocity relationship, as well as the acute responses and chronic adaptations to flywheel strength training, which have important implications for the relevance and trainability of the athletic population.

(b) Training dose: future studies are needed to clarify the physiological and physical benefits offered by different exposures of flywheel training in professional team sport athletes. This is particularly important considering the multifaceted nature of the training methodologies they are involved in, the congested fixture schedule and the limited time available for training in this specific sport population.

(c) Evidence-based guidelines: future studies are warranted to produce systematic guidelines about the methodological design and implementation of flywheel training. Researchers and practitioners necessitate clear recommendations about specific exercise aspects such as training periodization, training timing, exercise type (exercise orientation, length of muscle during the eccentric actions) exercise intensity (inertias), volume (sets and repetitions), rest interval, all possibly contributing to optimally enhance athletic performances. These aspects may be addressed by implementing well-designed randomized controlled trials (RCT) investigating the comparative effects of flywheel training and traditional resistance training methods both used in isolation or combined within an integrated approach.

(d) Studies design: the scientific literature clearly supports the utilization of flywheel training to induce adaptations in strength, muscle power, muscle mass, running speed, and sport-specific capabilities (Maroto-Izquierdo et al.,
2017; Nuñez Sanchez and Sáez de Villarreal, 2017; Petré et al., 2018). On the other hand, evidence in support of the superiority of flywheel training compared to traditional resistance training is not definitive in the current literature. Whereas Maroto-Izquierdo et al. (2017) suggested flywheel training as a preferable method as compared to traditional resistance training, contrasting results emerged from another meta-analysis (Vicens-Bordas et al., 2018). The latter included three RCTs and four non-RCTs that compared the two methodologies without finding statistically significant differences. Therefore, definitive evidence about the superiority of flywheel training needs to be confirmed, and this research topic should be further investigated with high quality study designs. As a consequence, we suggest practitioners to avoid a dichotomous approach and instead to integrate both flywheel and traditional resistance training in their daily routine.

(e) Monitoring and individualization: Another research direction worth perusing is the usefulness of individualizing the inertia intensity and the power output produced of flywheel exercises. Load quantification with rotatory encoders has recently been discussed but contradictory information is available mainly due to the inconsistency of used protocols and the variety of both flywheel machines and inertial loads, which question their reliability (Bollinger et al., 2018; Weakley et al., 2019). Further research on this topic could help to efficiently manage exercise prescription and monitoring especially in the frameworks of injury prevention, rehabilitation, and reconditioning where the applications of flywheel training are not currently well-explored. 
(f) Drawbacks of eccentric overload exercise: apart from their well-known and mentioned benefits, flywheel exercises are not completely free from risks or limitations. Flywheel exercises can induce a transient increase in local and circulating molecular markers of inflammation and muscle damage such as creatine kinase and lactate dehydrogenase after the first session (Fernandez-Gonzalo et al., 2014; Annibalini et al., 2019). This information is important as underlines the necessity of a progressive familiarization period, which can help reducing by products of muscle damage thus mitigating their negative impact on the following training sessions. Therefore, practitioners need to adopt a cautious approach preferring appropriate inertia load progression schemes with their athletes. This can be ensured through a few familiarization sessions in which lighter inertial loads (e.g., $0.03 \mathrm{~kg} \mathrm{~m}^{2}$ ) are progressively increased to greater ones (e.g., $\geq 0.06 \mathrm{~kg} \cdot \mathrm{m}^{2}$ ), particularly with novices and with athletes at high risk due to previous injury history or involved in return to sport programs.

\section{CONCLUSIONS}

This commentary provides practical recommendations about flywheel training in sports. The contemporary literature

\section{REFERENCES}

Annibalini, G., Contarelli, S., Lucertini, F., Guescini, M., Maggio, S., Ceccaroli, P., et al. (2019). Muscle and systemic molecular responses to a single flywheel based iso-inertial training session in resistance-trained men. Front. Physiol. 10:554. doi: 10.3389/fphys.2019.00554

Askling, C., Karlsson, J., and Thorstensson, A. (2003). Hamstring injury occurrence in elite soccer players after preseason strength training with eccentric overload. Scand. J. Med. Sci. Sport 13, 244-250. doi: 10.1034/j.1600-0838.2003.00312.x

Beato, M., Bigby, A. E. J., De Keijzer, K. L., Nakamura, F. Y., Coratella, G., and McErlain-Naylor, S. A. (2019a). Post-activation potentiation effect of eccentric overload and traditional weightlifting exercise on jumping and sprinting performance in male athletes. PLoS ONE 14:e0222466. doi: 10.1371/journal.pone.0222466

Beato, M., De Keijzer, K. L., Leskauskas, Z., Allen, W. J., Dello Iacono, A., and McErlain-Naylor, S. A. (2019b). Effect of postactivation potentiation after medium vs. high inertia eccentric overload exercise on standing long jump, countermovement jump, and change of direction performance. J. strength Cond. Res. doi: 10.1519/JSC.0000000000003214. [Epub ahead of print].

Beato, M., Madruga-Parera, M., Piqueras-Sanchiz, F., Moreno-Pérez, V., and Romero-Rodriguez, D. (2019c). Acute effect of eccentric overload exercises on change of direction performance and lower-limb muscle contractile function. J. Strength Cond. Res. doi: 10.1519/JSC.0000000000003359. [Epub ahead of print].

Beato, M., McErlain-Naylor, S. A., Halperin, I., and Dello Iacono, A. (2020). Current evidence and practical applications of flywheel eccentric overload exercises as postactivation potentiation protocols: a brief review. Int. J. Sports Physiol. Perform. 15, 154-161. doi: 10.1123/ijspp.2019-0476

Beato, M., Stiff, A., and Coratella, G. (2019d). Effects of postactivation potentiation after an eccentric overload bout on countermovement jump and lower-limb muscle strength. J. Strength Cond. Res. doi: 10.1519/JSC.0000000000003005. [Epub ahead of print].

Berg, H. E., and Tesch, A. (1994). A gravity-independent ergometer to be used for resistance training in space. Aviat. Space. Environ. Med. 65, 752-6.

Blazevich, A. J., and Babault, N. (2019). Post-activation potentiation versus postactivation performance enhancement in humans: historical perspective, suggests flywheel training as a valid alternative to traditional resistance training methods without clear evidence about its superiority among elite athletes. Flywheel exercise offers unique physiological responses compared to other resistance exercise modalities; therefore, practitioners should integrate both flywheel and traditional resistance training in their daily routine in order to optimize the benefits for their athletes. Future research is warranted to determine robust guidelines and construct objective consensus about the methodological aspects of flywheel training so to help researchers, practitioners and athletes for its implementation in their daily practice. In conclusion, this commentary supports the utilization of flywheel exercises implementation for both acute- and long-term athletic performance enhancements and provides researchers and practitioners with practical guidelines.

\section{AUTHOR CONTRIBUTIONS}

$\mathrm{MB}$ and $\mathrm{AD}$ have contributed equally to the paper.

\section{FUNDING}

No funding received from external bodies. Publication fees covered by University of Suffolk (UK). underlying mechanisms, and current issues. Front. Physiol. 10:1359. doi: 10.3389/fphys.2019.01359

Bollinger, L. M., Brantley, J. T., Tarlton, J. K., Baker, P. A., Seay, R. F., and Abel, M. G. (2018). Construct validity, test-retest reliability, and repeatability of performance variables using a flywheel resistance training device. J. Strength Cond. Res. doi: 10.1519/JSC.0000000000002647. [Epub ahead of print].

Boullosa, D., Del Rosso, S., Behm, D. G., and Foster, C. (2018). Post-activation potentiation (PAP) in endurance sports: a review. Eur. J. Sport Sci. 18, 595-610. doi: 10.1080/17461391.2018.1438519

Cermak, N. M., Snijders, T., McKay, B. R., Parise, G., Verdijk, L. B., Tarnopolsky, M. A., et al. (2013). Eccentric exercise increases satellite cell content in type II muscle fibers. Med. Sci. Sports Exerc. 45, 230-237. doi: 10.1249/MSS.0b013e318272cf47

Colliander, E. B., and Tesch, P. A. (1990). Effects of eccentric and concentric muscle actions in resistance training. Acta Physiol. Scand. 140, 31-39. doi: 10.1111/j.1748-1716.1990.tb08973.x

Coratella, A. G., Beato, M., Cè, E., Scurati, R., and Milanese, C. (2019). Effects of inseason enhanced negative work-based vs traditional weight training on change of direction and hamstrings-to-quadriceps ratio in soccer players. Biol. Sport 36, 241-248. doi: 10.5114/biolsport.2019.87045

Cuenca-Fernández, F., López-Contreras, G., and Arellano, R. (2015). Effect on swimming start performance of two types of activation protocols: lunge and YoYo squat. J. strength Cond. Res. 29, 647-655. doi: 10.1519/JSC.0000000000000696

Cuenca-Fernández, F., López-Contreras, G., Mourão, L., de Jesus, K., de Jesus, K., Zacca, R., et al. (2019). Eccentric flywheel post-activation potentiation influences swimming start performance kinetics. J. Sports Sci. 37, 443-451. doi: $10.1080 / 02640414.2018 .1505183$

Cuenca-Fernández, F., Ruiz-Teba, A., López-Contreras, G., and Arellano, R. (2018). Effects of 2 types of activation protocols based on postactivation potentiation on $50-\mathrm{m}$ freestyle performance. J. strength Cond. Res. doi: 10.1519/JSC.0000000000002698. [Epub ahead of print].

de Hoyo, M., de la Torre, A., Pradas, F., Sañudo, B., Carrasco, L., MateoCortes, J., et al. (2014). Effects of eccentric overload bout on change of direction and performance in soccer players. Int. J. Sports Med. 36, 308-314. doi: $10.1055 / \mathrm{s}-0034-1395521$ 
de Hoyo, M., Pozzo, M., Sañudo, B., Carrasco, L, Gonzalo-Skok, O., Domínguez-Cobo, S., et al. (2015). Effects of a 10-week in-season eccentricoverload training program on muscle-injury prevention and performance in junior elite soccer players. Int. J. Sports Physiol. Perform. 10, 46-52. doi: 10.1123/ijspp.2013-0547

de Keijzer, K. L., McErlain-Naylor, S. A, Dello Iacono, A., and Beato, M. (2020). Effect of volume on eccentric overload-induced postactivation potentiation of jumps. Int. J. Sports Physiol. Perform. 1-6. doi: 10.1123/ijspp.2019-0411. [Epub ahead of print].

Dello Iacono, A., Beato, M., and Halperin, I. (2019). The effects of clusterset and traditional-set postactivation potentiation protocols on vertical jump performance. Int. J. Sports Physiol. Perform. 1-6. doi: 10.1123/ijspp.2019-0186. [Epub ahead of print].

Douglas, J., Pearson, S., Ross, A., and McGuigan, M. (2017). Eccentric exercise: physiological characteristics and acute Responses. Sport. Med. 47, 663-675. doi: 10.1007/s40279-016-0624-8

Douglas, J., Pearson, S., Ross, A., and McGuigan, M. (2018). Effects of accentuated eccentric loading on muscle properties, strength, power, and speed in resistance-trained rugby players. J. Strength Cond. Res. 32, 2750-2761. doi: 10.1519/JSC.0000000000002772

Dudley, G. A., Tesch, P. A., Miller, B. J., and Buchanan, P. (1991). Importance of eccentric actions in performance adaptations to resistance training. Aviat. Space. Environ. Med. 62, 543-50.

Fernandez-Gonzalo, R., Lundberg, T. R., Alvarez-Alvarez, L., and de Paz, J. A. (2014). Muscle damage responses and adaptations to eccentric-overload resistance exercise in men and women. Eur. J. Appl. Physiol. 114, 1075-1084. doi: 10.1007/s00421-014-2836-7

Franchi, M. V., and Maffiuletti, N. A. (2019). Distinct modalities of eccentric exercise: different recipes, not the same dish. J. Appl. Physiol. 127, 881-883. doi: 10.1152/japplphysiol.00093.2019

Hody, S., Croisier, J-L., Bury, T., Rogister, B., and Leprince, P. (2019). Eccentric muscle contractions: risks and benefits. Front. Physiol. 10:536. doi: 10.3389/fphys.2019.00536

Illera-Domínguez, V., Nuell, S., Carmona, G., Padullés, J. M., Padullés, X., Lloret, M., et al. (2018). Early functional and morphological muscle adaptations during short-term inertial-squat training. Front. Physiol. 9:1265. doi: $10.3389 /$ fphys.2018.01265

Maroto-Izquierdo, S., Fernandez-Gonzalo, R., Magdi, H. R., ManzanoRodriguez, S., González-Gallego, J., De Paz, J., et al. (2019). Comparison of the musculoskeletal effects of different iso-inertial resistance training modalities: flywheel vs. electric-motor. Eur. J. Sport Sci. 19, 1184-1194. doi: 10.1080/17461391.2019.1588920

Maroto-Izquierdo, S., García-López, D., Fernandez-Gonzalo, R., Moreira, O. C., González-Gallego, J., and de Paz, J. A. (2017). Skeletal muscle functional and structural adaptations after eccentric overload flywheel resistance training: a systematic review and meta-analysis. J. Sci. Med. Sport 20, 943-951. doi: 10.1016/j.jsams.2017.03.004

Martinez-Aranda, L. M., and Fernandez-Gonzalo, R. (2017). Effects of inertial setting on power, force, work, and eccentric overload during flywheel resistance exercise in women and men. J. Strength Cond. Res. 31, 1653-1661. doi: 10.1519/JSC.0000000000001635

Moore, D. R., Phillips, S. M., Babraj, J. A., Smith, K., and Rennie, M. J. (2005). Myofibrillar and collagen protein synthesis in human skeletal muscle in young men after maximal shortening and lengthening contractions. Am. J. Physiol. Endocrinol. Metab. 288, E1153-E1159. doi: 10.1152/ajpendo.00387.2004

Norrbrand, L., Fluckey, J. D., Pozzo, M., and Tesch, P. A. (2008). Resistance training using eccentric overload induces early adaptations in skeletal muscle size. Eur. J. Appl. Physiol. 102, 271-281. doi: 10.1007/s00421-007-0583-8

Nuñez Sanchez, F. J., and Sáez de Villarreal, E. (2017). Does flywheel paradigm training improve muscle volume and force? a meta-analysis. J. Strength Cond. Res. 31, 3177-3186. doi: 10.1519/JSC.0000000000002095

Petré, H., Wernstål, F., and Mattsson, C. M. (2018). Effects of flywheel training on strength-related variables: a meta-analysis. Sport. Med. Open 4:55. doi: 10.1186/s40798-018-0169-5

Roig, M., O’Brien, K., Kirk, G., Murray, R., McKinnon, P., Shadgan, B, et al. (2009). The effects of eccentric versus concentric resistance training on muscle strength and mass in healthy adults: a systematic review with meta-analysis. Br. J. Sports Med. 43, 556-568. doi: 10.1136/bjsm.2008.051417

Sabido, R., Hernández-Davó, J. L., and Pereyra-Gerber, G. T. (2018). Influence of different inertial loads on basic training variables during the flywheel squat exercise. Int. J. Sports Physiol. Perform. 13, 482-489. doi: 10.1123/ijspp.2017-0282

Suarez-Arrones, L., Saez de Villarreal, E., Núñez, F. J., Di Salvo, V., Petri, C., Buccolini, A., et al. (2018). In-season eccentric-overload training in elite soccer players: effects on body composition, strength and sprint performance. PLoS ONE 13:e0205332. doi: 10.1371/journal.pone.0205332

Suchomel, T. J., Nimphius, S., Bellon, C. R., and Stone, M. H. (2018). The importance of muscular strength: training considerations. Sport Med. 48, 765-785. doi: 10.1007/s40279-018-0862-Z

Tesch, P. A., Fernandez-Gonzalo, R., and Lundberg, T. R. (2017). Clinical applications of iso-inertial, eccentric-overload $\left(\mathrm{YoYo}^{\mathrm{TM}}\right.$ ) resistance exercise. Front. Physiol. 8:241. doi: 10.3389/fphys.2017.00241

Tillin, N. A., and Bishop, D. (2009). Factors modulating post-activation potentiation and its effect on performance of subsequent explosive activities. Sports Med. 39, 147-166. doi: 10.2165/00007256-200939020-00004

Toigo, M., and Boutellier, U. (2006). New fundamental resistance exercise determinants of molecular and cellular muscle adaptations. Eur. J. Appl. Physiol. 97, 643-663. doi: 10.1007/s00421-006-0238-1

Tous-Fajardo, J., Gonzalo-Skok, O., Arjol-Serrano, J. L., and Tesch, P. (2016). Enhancing change-of-direction speed in soccer players by functional inertial eccentric overload and vibration training. Int. J. Sports Physiol. Perform. 11, 66-73. doi: 10.1123/ijspp.2015-0010

Tous-Fajardo, J., Maldonado, R. A., Quintana, J. M., Pozzo, M., and Tesch, P. A. (2006). The flywheel leg-curl machine: offering eccentric overload for hamstring development. Int. J. Sports Physiol. Perform. 1, 293-298. doi: 10.1123 /ijspp.1.3.293

Vicens-Bordas, J., Esteve, E., Fort-Vanmeerhaeghe, A., Bandholm, T., and Thorborg, K. (2018). Is inertial flywheel resistance training superior to gravity-dependent resistance training in improving muscle strength? a systematic review with meta-analyses. J. Sci. Med. Sport 21, 75-83. doi: 10.1016/j.jsams.2017.10.006

Wagle, J. P., Taber, C. B., Cunanan, A. J., Bingham, G. E., Carroll, K. M., DeWeese, B. H., et al. (2017). Accentuated eccentric loading for training and performance: a review. Sports Med. 47, 2473-2495. doi: 10.1007/s40279-0170755-6

Wallace, B. J., Shapiro, R., Wallace, K. L., Abel, M. G., and Symons, T. B. (2019). Muscular and neural contributions to postactivation potentiation. J. strength Cond. Res. 33, 615-625. doi: 10.1519/JSC.0000000000003011

Weakley, J., Fernández-Valdés, B., Thomas, L., Ramirez-Lopez, C., and Jones, B. (2019). Criterion validity of force and power outputs for a commonly used flywheel resistance training device and bluetooth app. J. Strength Cond. Res. 33, 1180-1184. doi: 10.1519/JSC.0000000000003132

Wernbom, M., Augustsson, J., and Thome, R. (2007). The influence of frequency, intensity, volume and mode of strength training on whole muscle cross-sectional area in humans. Sport Med. 37, 225-264. doi: 10.2165/00007256-200737030-00004

Zamparo, P., Bolomini, F., Nardello, F., and Beato, M. (2015). Energetics (and kinematics) of short shuttle runs. Eur. J. Appl. Physiol. 115, 1985-1994. doi: 10.1007/s00421-015-3180-2

Conflict of Interest: The authors declare that the research was conducted in the absence of any commercial or financial relationships that could be construed as a potential conflict of interest.

The handling editor declared past co-authorship with one of the authors, AD.

Copyright (C) 2020 Beato and Dello Iacono. This is an open-access article distributed under the terms of the Creative Commons Attribution License (CC BY). The use, distribution or reproduction in other forums is permitted, provided the original author(s) and the copyright owner(s) are credited and that the original publication in this journal is cited, in accordance with accepted academic practice. No use, distribution or reproduction is permitted which does not comply with these terms. 